\title{
Síntesis intramedular encerrrojada en las fracturas del extremo distal del radio
}

\author{
R. Delgado Velilla ${ }^{(1)}$, J. Martínez Renobales ${ }^{2)}$, J. A. Tovar Gallego ${ }^{(1)}$ \\ UNIDAD DE CIRUGÍA DE LA MANO \\ ${ }^{(1)}$ CLÍNICA LA MILAGROSA, MADRID \\ (2)PolicLÍNICA GUIPÚZCOA, SAN SEBASTIÁN
}

Correspondencia:

Dr. Rafael Delgado Velilla

Clínica La Milagrosa

Servicio de Traumatología y Cirugía Ortopédica

Unidad de Cirugía de la Mano

C/ Modesto Lafuente, 14 - 28010 Madrid

Tel.: 914472100

e-mail.rdelgadovelilla@gmail.com

Introducción: Las fracturas del extremo distal del radio, son una de las patologías mas frecuentes de la extremidad superior. Fundamentalmente afectan a dos grupos poblacionales bien diferenciados. Un primer grupo, el más numeroso, correspondería a mujeres posmenopáusicas, y un segundo grupo a jóvenes de cualquier sexo que practican deporte habitualmente. El tratamiento de estas lesiones, debido a la alta agresividad quirúrgica de las síntesis extramedulares, sean estas dorsales y/o volares, implica un excesivo número de tratamientos incruentos, no siempre con el resultado funcional debido. Material y Método: Presentamos, además de la técnica quirúrgica específica de la síntesis intramedular encerrojada, una serie de treinta y siete fracturas en treinta y seis enfermos, que fueron tratados con dicho método. Esta técnica, presenta una mínima agresividad quirúrgica y un resultado funcional muy bueno, acortando los tiempos medios de incapacidad en parámetros significativos.

Resultados: En la serie que presentamos, todos los enfermos han consolidado sus fracturas en menos de ocho semanas, con tiempos de inmovilización postquirúrgica mínimos, una recuperación funcional excelente y una magnífica aceptación por parte de los accidentados.

Conclusiones: La síntesis intramendular encerrojada de las fracturas del extremo distal del radio, por la rápida recuperación de los enfermos, la baja agresividad quirúrgica y las buenas condiciones postquirúrgicas obtenidas, constituye una técnica alternativa de tratamiento muy válida para este tipo de lesiones.

Palabras Clave: Fracturas radio. Síntesis intramedular. Micronail ${ }^{\circledR}$. Radio distal.
Introduction: The distal radius fractures, are one of most frequent in the traumatic pathology of upper extremity. Principally, they affect to two different groups. The first group, belongs more often to post menopause women, and the second groupe, belongs to young sportmen of both sex. Due to the high surgery agressivity of the extramedular synthesis, dorsal or volar approach, the treatment of this kina of fractures, produce an excessive number of orthopaedic treatments not always with satisfactory functional results.

Materials and methods: We present a serie of 37 fractures in 36 patients treated with intramedular distal radius synthesis. This technique present a low surgery agressivity, and a very good functional result, reducing "at minimun" the medium times of incapacity in significative parameters.

Results: In the serie that we present, all patients obtain complete consolidation of their fractures in short time, without post surgery immobilisation time, an excellent functional recovery and a great satisfaction of patients.

Conclusions: The intramedular synthesis of distal radius, is a very good indicated technique in this pathologic group due to the phase recovery of patients, low surgery agressivity and good post surgery conditions.

Keywords: Radius fractures. Intramedular synthesis. Micronail ®. Distal Radius. 


\section{INTRODUCCIÓN}

Las fracturas del extremo distal del radio, son una de las lesiones más frecuentes que se observan en la extremidad superior. Su prevalencia tiene un alto coste en la sociedad, tanto desde el punto de vista económico como desde la propia situación de los pacientes afectos de esta patología, ya que deben soportar en la mayoría de los casos, tratamientos que conllevan una inmovilización prolongada y una rehabilitación persistente, así como presentar una frecuencia no desdeñable de secuelas, fundamentalmente la pérdida de movilidad y la disminución de la fuerza de prensión.

La mayor incidencia de estas fracturas se produce en dos grupos diferenciados. En el primer grupo, hablamos de mujeres postmenopáusicas, englobando esta patología en el grupo de fracturas osteoporóticas, con un resultado funcional mas incierto todavía, debido en gran parte al conformismo en los resultados a causa de la edad de las pacientes, lo que incita a una escasa actuación terapéutica, optando en la mayoría de los casos por un tratamiento incruento en fracturas que podrían solucionarse de una manera mucho mejor con tratamientos quirúrgicos poco agresivos. En el segundo grupo, el de jóvenes deportistas a los que se les puede añadir aquellas personas con accidentes laborales y/o de tráfico, independientemente del género, las fracturas suelen ser de alta energía, con una gravedad pronóstica mucho mayor, en las cuales se establecen todos los métodos terapéuticos posibles para la correcta solución del trauma. Entre éstos, la reducción y síntesis temporal, la síntesis abierta por vía volar o dorsal y los fijadores externos, compendian los mecanismos de actuación predominantes.

Entendemos que la síntesis intramedular encerrojada, un nuevo concepto en el tratamiento de estas fracturas, es un método que, a priori, es ideal para el tratamiento de las mismas, aportando una rápida movilización de la muñeca, una recuperación funcional eficaz y una reincorporación del enfermo a sus actividades diarias mucho mas breve que con los métodos tradicionales.

\section{MATERIAL Y MÉTODO}

Presentamos una serie de treinta y siete fracturas en treinta y seis pacientes intervenidos con síntesis intramedular. La edad media de estos pacientes fue de 54 años, con un rango de 28 a 75 años. El estudio se realizó durante el periodo comprendido entre el 2005 y el 2006, con un tiempo medio de evolución de once semanas, con un rango entre las 7 y las 18 semanas. En nuestra serie, veintiséis enfermos eran mujeres y diez varones, predominando el lado izquierdo en proporción de 22 a 13. Uno de los casos fue bilateral. Como lesiones asociadas encontramos una fractura de cúbito distal, tratada con síntesis temporal con agujas de Kischnner, una fractura de escafoides, en la que utilizamos un tornillo para síntesis percutánea y una rotura del extensor pollicis longus (EPL) en el derecho que requirió plastia tendinosa, teniendo un resultado funcional completo, tanto de la función extensora del primer dedo como de la fractura intervenida.

Para la clasificación de las fracturas, utilizamos la propuesta por Frykman, de amplia aceptación y muy significativa para valorar la afectación articular en estas fracturas ${ }^{1}$. Así pues y por tipo de fractura, ocho correspondieron al tipo 1 de la clasificación de Frykman, cinco al 2 , cuatro al tipo 3 , tres al 4 , seis al tipo 5 , seis al tipo 7 y cinco al 8 .

La actuación quirúrgica en las fracturas de radio distal tiene unos criterios anatómicos y funcionales basados en la obtención de la consolidación, evitando las secuelas y obteniendo los mejores resultados funcionales posibles. Así, el simple hecho de evitar la inmovilización posquirúrgica, con la consecuente ganancia funcional desde los primeros días, y la ausencia de algodistrofia o rigidez, sería ya un criterio quirúrgico. En cuanto a la radiología, consideramos como criterios inexcusablemente quirúrgicos, tras intentar la reducción por manipulación, la inestabilidad de la reducción, el colapso radial, un escalón articular superior a $0,2 \mathrm{~cm}$., que podría tolerarse hasta 0 '5 en personas de edad avanzada o con gran fragilidad ósea por porosis, y un desplazamiento articular superior a $0,7 \mathrm{~cm}$., ya que la experiencia nos demuestra que desplazamientos en la línea articular inferiores a estas cifras, si no producen resalte, cuando la movilización es precoz, no crean secuelas significativas.

La síntesis intramedular de estas fracturas está indicada, como ya se ha mencionado, en 


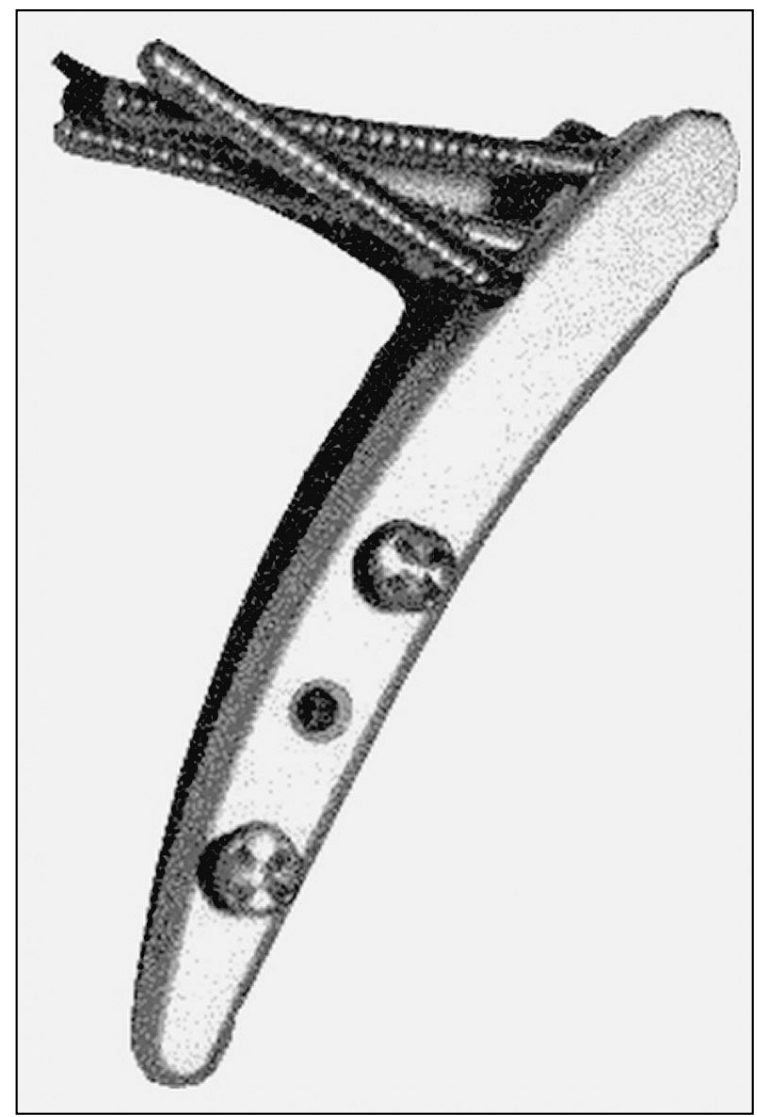

Figura 1: Micronail ®.

todas aquellas en las que se consiga y mantenga, aún con síntesis temporales o sistemas de tracción, una correcta reducción durante el acto quirúrgico. También es necesario que el fragmento estiloideo, donde vamos a introducir el implante, tenga tamaño suficiente para conseguir la necesaria estabilidad y permita la realización de la osteotomía. No se realizó inmovilización posquirúrgica en ningún caso, comenzando de manera inmediata con movimientos activos de muñeca, salvo en el caso de la fractura de cúbito asociada, que se mantuvo durante 4 semanas y en la sección del EPL, que se mantuvo durante 3 semanas.

La duración del tratamiento, desde la cirugía hasta el alta fue de 10 semanas de media.

\section{TÉCNICA QUIRÚRGICA}

La técnica de enclavijamiento intramedular en fracturas de radio distal es una técnica muy sistematizada, de fácil aplicación mientras se mantenga el correcto orden de los pasos quirúr- gicos y se tenga una noción clara de la biomecánica de estas fracturas.

Esta técnica está indicada en todas aquellas fracturas en las que se pueda obtener una correcta reducción de la fractura previa a la cirugía, corrección que debemos mantener en el acto quirúrgico, ya sea por síntesis temporales, tracción mantenida o cualquier otro método que sea eficaz para mantener la reducción. El número de fragmentos no es importante para la indicación siempre que el fragmento estiloideo sea de tamaño suficiente para la introducción del implante ${ }^{2}$.

Utilizamos la síntesis intramedular diseñada por Wright y denominada Micronail $₫$ (Wright Medical Technology, Inc., Arlington, USA), fabricada en titanio y con forma curvada, que permite una correcta adaptación al canal medular del radio (Figura 1). Se acompaña de una instrumentación muy sistematizada, que acarrea pocos problemas a la hora del implante. Esta técnica ha sido experimentada por los Drs. Slade, Tan, Capo y Warburton, en Estados Unidos y es la única serie que encontramos publicada ${ }^{2}$.

El enfermo se coloca en decúbito supino, con el miembro afecto sobre mesa de mano translúcida y con control radioscópico durante toda la intervención. Una vez realizada la reducción y mantenida con el método más eficaz, se realiza el abordaje distal. La incisión se practica en la estiloides radial, en el segundo compartimento extensor, paralela al radio y de una extensión de 2-3 cms. Hay que identificar y proteger la rama sensitiva del nervio radial que aparece sistemáticamente en el abordaje (Figura 2). Una vez abordado el radio y desperiostiza-

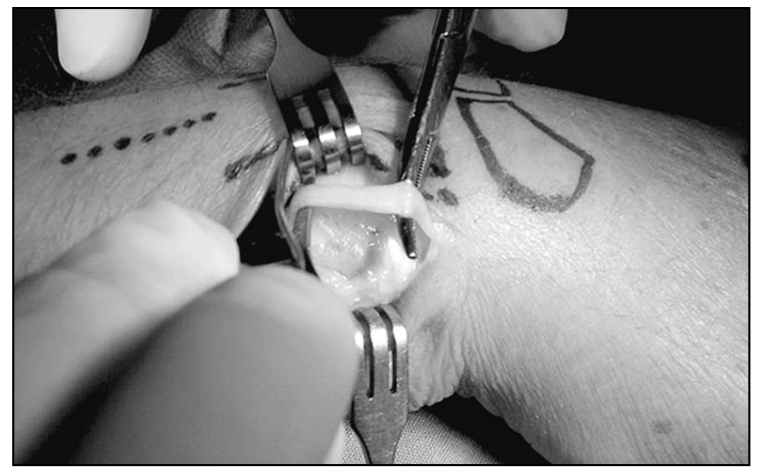

Figura 2: Identificación de la rama sensitiva del nervio radial antes de proceder a la osteotomía ovoide del radio. 


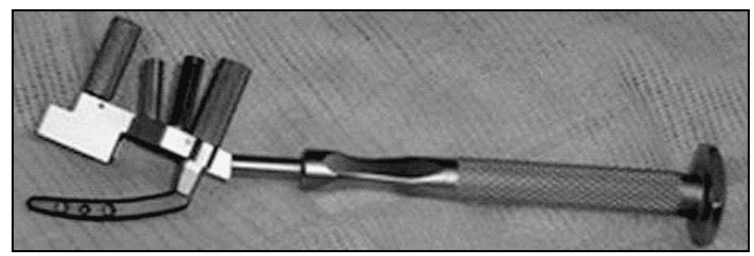

Figura 3: Guía del implante con el Micronail@ a punto de ser insertado.

da la estiloides en una extensión de $1 \mathrm{~cm}$. se realiza, siempre bajo control radioscópico, la introducción de una aguja de Kischnner sobre la guía que presenta a tal efecto la instrumentalización. Dicha guía debe introducirse en el canal medular paralela a la diafisis del radio y con una inclinación de $45^{\circ}$ sobre ella. Una vez introducida la aguja, y sobre ella, se realiza la osteotomía con broca canulada, que al mantener la dirección de la aguja realiza una osteotomía ovoiforme, que permite el paso del implante. En caso necesario, esta osteotomía puede ampliarse mediante una gubia, evitando el exceso de apertura de la cortical.

A través de la osteotomía se introduce el iniciador, con forma idéntica a la del implante, que nos marcará la dirección de éste y nos permitirá comprobar el correcto direccionamiento del mismo.

A partir de éste momento, iniciaremos el fresado del canal medular con las fresas numeradas, que nos darán el tamaño del implante adecuado a cada canal y para cada fractura. Una vez determinado el tamaño del implante, montaremos la guía con el implante definitivo (Figura 3), el cual introduciremos a través de la osteotomía hasta posicionarlo correctamente.

Este montaje permite la introducción de los tornillos distales a través de las guías correspondientes. Estos tornillos, en número de tres, estabilizan los fragmentos y mantienen la fractura en posición anatómica. El primer tornillo a introducir, el mas distal, debe ir paralelo a la superficie articular del radio, a una distancia máxima de $1,5 \mathrm{~cm}$. de la misma. Los otros dos tornillos, convergentes entre sí en el plano antero posterior y divergentes en dorsal/volar, mantienen el resto de la estructura en su posición. Los tornillos distales son de compresión y roscados al implante, lo que permite realizar su función de manera eficaz, manteniendo al mismo tiempo, la estabilidad necesaria.

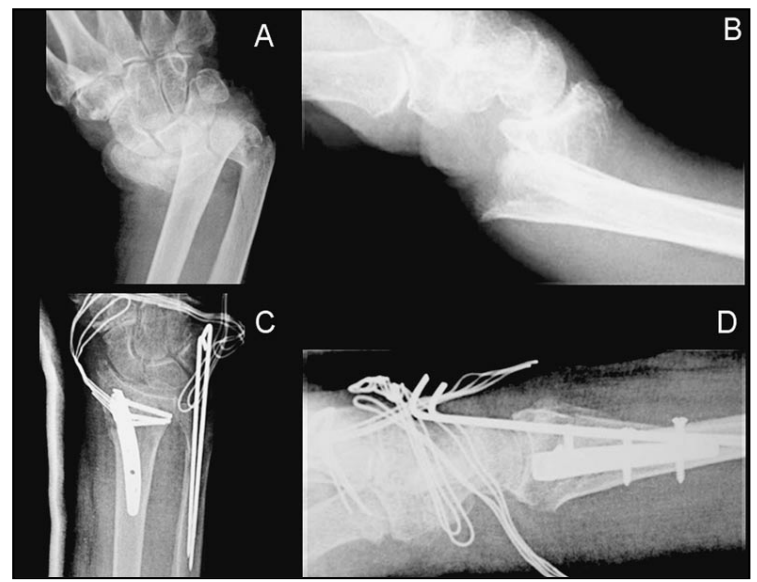

Figura 4: Fractura conminuta intraarticular del extremo distal del radio tratada con síntesis interna con $\mathrm{Mi}$ cronail ${ }^{\circledR}$. A y B) Radiografías iniciales anteroposterior y lateral, respectivamente. $C$ y D) Radiografías postoperatorias anteroposterior y lateral, respectivamente.

Una vez colocados los tornillos distales y sobre la misma guía, se posiciona la plantilla de los tornillos proximales, roscados a la diafisis, bicorticales y que mantienen, tanto la estabilidad de la fractura como la correspondiente longitud del radio. Para esto, a través del orificio que presenta la plantilla, y tras la tracción adecuada, se introduce una aguja de Kischnner que mantiene la correcta relación radiocubital, evitando las minusvalías radiales. La plantilla permite la introducción percutánea de los tornillos proximales a través de las guías para broca y tornillos.

Con los tornillos proximales en su posición podemos retirar la aguja de Kischnner que mantiene la distancia radial, dando por terminada la cirugía Debemos comprobar con la escopia la correcta estabilidad de la fractura, realizando el arco articular completo y comprobando que los fragmentos permanecen inmóviles (Figura 4).

No es necesaria la inmovilización postquirúrgica, poniendo un vendaje blando para evitar edemas y comenzando con la movilización en los primeros días.

\section{RESULTADOS}

Valoramos los resultados basándonos en dos tipos de criterios. El primero de ellos, determina el resultado radiológico, valorando la consolidación de la fractura, la relación radiocubi- 
tal distal, los desplazamientos dorsales o volares residuales y los signos radiológicos de inestabilidad carpiana no presentes previamente, mientras que el segundo criterio, valora los resultados funcionales al alta siguiendo los parámetros habitualmente utilizados, como son los arcos de movilidad, la fuerza de prensión y la satisfacción personal del paciente. La valoración global la realizamos, calculando la media de las puntuaciones de cada parámetro, aplicando un baremo de gradación en: Excelente (de 2,6 a 3 puntos), Bueno (de 1,6 a 2,5 puntos, Regular (de 1 a 1,5 puntos) y Malo (de 0 a 1 puntos) (Tabla I).

\section{Tabla I - Valoración de los resultados}

- Flexión y extensión:

0 Ausencia de flexión

1 Entre $10^{\circ}$ y $40^{\circ}$

2 Entre $41^{\circ}$ y $70^{\circ}$

3 Más de $70^{\circ}$

- Lateralizaciones, pronosupinación y fuerza de prensión

0 Ausencia de actividad

1 Menos del $30 \%$ de la muñeca contralateral

2 Entre $30 \%$ y $70 \%$ de la contralateral

3 Mas del $70 \%$

- Deformidad del carpo:

0 Deformidad evidente que limita la función

1 Deformidad evidente que no afecta la función

2 Deformidad leve/moderada

3 Sin deformidad

- Satisfacción del paciente:

0 Ninguna satisfacción

1 Poca satisfacción

2 Satisfecho con el resultado

3 Muy satisfecho con el resultado

0 a 1: Resultado malo; 1 a 1,5: resultado regular; 1,6 a 2,5: Resultado bueno; 2,6 a 3: Resultado excelente.

En función de los criterios de valoración seguidos, obtuvimos un $67,56 \%$ (25) de casos excelentes, $24,32 \%$ (9) de buenos, $8,1 \%$ (3) regulares y ninguno malo. El tiempo medio de alta, con los resultados funcionales expuestos, fue

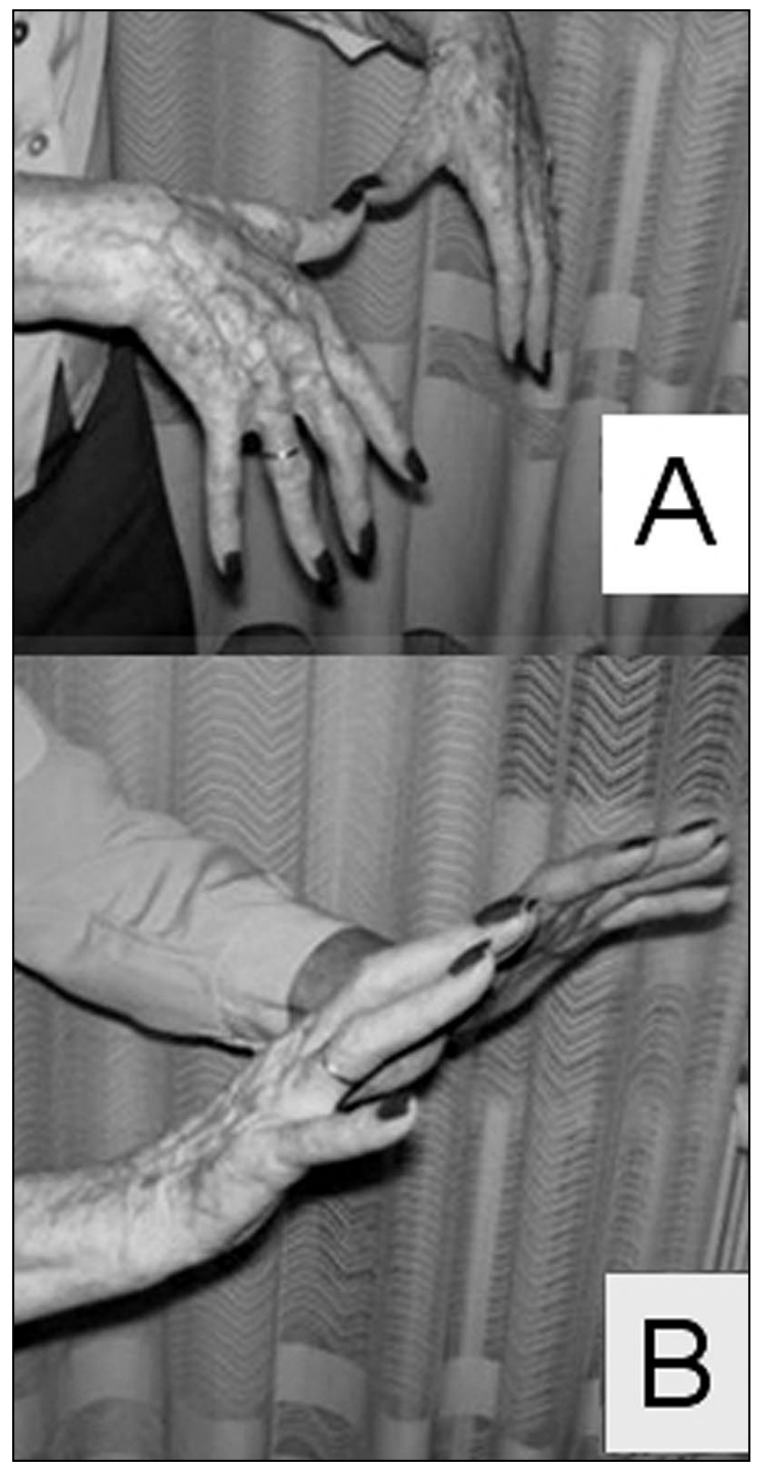

Figura 5: Resultado funcional. A) Flexión. B) Extensión.

de 10 semanas (Figura 5). Todos los pacientes se incorporaron normalmente a sus ocupaciones habituales.

\section{DISCUSIÓN}

Las fracturas de la extremidad distal del radio son habitualmente tributarias de tratamientos incruentos ${ }^{3}$. Esto es debido a que los métodos quirúrgicos actuales, implican, tanto en abordajes dorsales como volares, una alta agresión quirúrgica, con tiempos de recuperación francamente altos y con elevado número de secuelas, a pesar de los nuevos materiales dispo- 
nibles, con placas de bajo perfil y de metales mas idóneos. Asimismo, es fundamental tratar las patologías asociadas del cúbito pues de estas va a depender, en gran medida, el resultado final $^{4-6}$. Los métodos menos agresivos, como son los fijadores externos, síntesis temporales con agujas de Kischnner o yesos bipolares, mantienen los tiempos de inmovilización habituales pero no siempre obtienen los resultados apeteci$\operatorname{dos}^{7-9}$, mientras que los métodos combinados de fijación interna y externa, utilizados con cierta frecuencia hace años, están siendo cada vez menos empleados por su escasa aportación al resultado final respecto a los métodos tradicionales $^{10}$. En cuanto a los tratamientos incruentos con reducción cerrada de la fractura e inmovilización con vendaje enyesado, no consiguen en la mayoría de los casos, el mantenimiento de la reducción, siendo habitual la impactación del radio a los pocos días. La primera consecuencia de esta impactación es la minusvalía radial, con la deformidad evidente de la muñeca y los trastornos funcionales que ello acarrea. Las desviaciones fragmentarias en dorsal y volar tras la reducción son, con mayor frecuencia de la deseada, aceptadas, sobre todo en aquellos casos en que el paciente tiene una edad avanzada.

El tratamiento que se propone en este trabajo, es decir, la síntesis intramedular encerroja- da, es un concepto nuevo dentro del abanico de métodos quirúrgicos utilizados para tal fin. Sin embargo, debido a su escasa agresividad quirúrgica y al poco tiempo de inmovilización, está indicada en cualquier edad, iniciando la movilidad en etapas muy tempranas, incluso inmediatamente posteriores a la cirugía, con lo que el tiempo global de tratamiento se acorta substancialmente. Al no realizar abordajes articulares, no añadimos causas de rigidez a las que ya de por sí presenta la fractura, evitando adherencias y fibrosis. También es mucho menor, por la misma causa, la aparición de algodistrofias. Asimismo, con este método, aumentamos de manera significativa el número de fracturas tributarias de tratamiento quirúrgico, restamos riesgo a las técnicas abiertas y conseguimos resultados mucho más satisfactorios y en menos tiempo que los tratamientos actuales, incluidos los incruentos. Todas estas causas conllevan a una satisfacción amplia del paciente ante su terapia, un coste mucho menor del tratamiento y una incorporación mas rápida a sus ocupaciones habituales.

Por todo lo anteriormente expuesto, consideramos que la técnica del enclavado intramedular encerrojado en las fracturas del extremo distal del radio, constituye una alternativa de tratamiento segura y eficaz.

\section{BIBLIOGRAFÍA}

1. Frykman G. Fracture of the distal radius including sequelaeshoulder-hand-finger syndrome, disturbance in the distal radioulnar joint and impairment of nerve function. A clinical and experimental study. Acta Orthop Scand, 1967; Suppl 108: 3.

2. Tan V, Capo J, Warburton M: Distal radius fracture fixation with an intramedullary nail. Tech Hand Up Extr Surg, 2005; 9: 195-201.

3. Goldfarb CA, Rodzki JR, Catalana LW, Hugues M, Borreli J Jr: Fifteen-Years outcome of displaced intra-articular fractures of the distal radius. J. Hand Surg Am, 2006; 31: 633-9.

4. Fernandez F, Benrahho J: Treatment of dorsally displaced dis- tal radius fractures with a double dorsal plate: A study of 12 patients. Chir Main, 2005; 25 : 27-32.

5. Barrie KA, Wolf SW: Internal fixation for intra-articular distal radius fractures. Tech Hand Up Extrem Surg, 2002; 6:1020.

6. De Pedro JA, García-Navarrete F, De Lucas F, Otero R, Oteo A, López-Durán Stern L: Internal fixation of ulna fractures by locking nail. Clin Orthop, 1992; 283: 81-5.

7. Ring D, Júpiter JB, Brennwald J, Büchler U, Hastings H: Prospective multicenter trial of a plate for dorsal fixation of distal radius fractures. J. Hand Surg Am, 1997; 22: 277-284.
8. Nakata RY, Chand Y, Matiko JD, Frykman GK, Wood VE: External fixators for wrist fractures: a biomechanical and clinical study. J. Hand Surg Am, 1985; 10: 845-851.

9. Bishay M, Aguilera X, Grant J, Dunkerley DR: The results of external fixation of the radius in the treatment of comminute intra-articular fractures of the distal end. J Hand Surg Br, 1994; 19: 378-383.

10. Jebson PJ, Blair WF: Combined Internal and External fixation of complex intra-articular radius fractures using dorsal and volar approaches. Tech Hand Up Extrem. Surg, 2003; 4:161-6. 